\title{
Sectional split extensions arising from lifts of groups
}

\author{
Rok Požar \\ Inštitut za matematiko, fiziko in mehaniko, Jadranska 19, 1000 Ljubljana, Slovenia \\ and \\ Fakulteta za matematiko, naravoslovje in informacijske tehnolgije, Univerza na \\ Primorskem, Glagoljaška 8, 6000 Koper, Slovenia
}

Received 3 September 2012, accepted 4 April 2013, published online 9 September 2013

\begin{abstract}
Covering techniques have recently emerged as an effective tool used for classification of several infinite families of connected symmetric graphs. One commonly encountered technique is based on the concept of lifting groups of automorphisms along regular covering projections $\wp: \tilde{X} \rightarrow X$. Efficient computational methods are known for regular covers with cyclic or elementary abelian group of covering transformations $\mathrm{CT}(\wp)$.

In this paper we consider the lifting problem with an additional condition on how a group should lift: given a connected graph $X$ and a group $G$ of its automorphisms, find all connected regular covering projections $\wp: \tilde{X} \rightarrow X$ along which $G$ lifts as a sectional split extension. By this we mean that there exists a complement $\bar{G}$ of $\mathrm{CT}(\wp)$ within the lifted group $\tilde{G}$ such that $\bar{G}$ has an orbit intersecting each fibre in at most one vertex. As an application, all connected elementary abelian regular coverings of the complete graph $K_{4}$ along which a cyclic group of order 4 lifts as a sectional split extension are constructed.
\end{abstract}

Keywords: Covering projection, graph, group extension, lifting automorphisms, voltage assignment. Math. Subj. Class.: 05C50, 05E18, 20B40, 20B25, 20K35, 57M10

\section{Introduction}

Graph covers play a significant role when symmetry properties of graphs are investigated. One of the commonly used techniques is based on the concept of lifting automorphisms along regular covering projections. Applications of this technique have been used to classify families of graphs with given structural properties (see for instance $[2,11,12,19,20]$ ).

E-mail address: pozar.rok@gmail.com (Rok Požar) 
In its most general form the problem of lifting automorphisms is well understood. Much attention has been devoted to finding the necessary and sufficient lifting conditions in combinatorial terms, see $[15,16,26,27]$. Nevertheless, these general results are rather hopeless to apply when concrete examples and more detailed questions related to symmetry properties of graphs are considered.

In a more specific setting of regular covers in which the group of covering transformations is either cyclic or elementary abelian, the situation changes. For such covers, efficient computational methods are known. For example, in the case of elementary abelian regular covers, the idea behind the approach developed in [19] is to reduce the general lifting problem to that of finding invariant subspaces of matrix groups over prime fields, linearly representing the action of automorphisms on the first homology group of the graph. Applying this method to a number of symmetric graphs - including the complete graphs $K_{4}$ [20] and $K_{5}$ [13], the Möbius-Kantor graph [18], the complete bipartite graph $K_{3,3}$ [20], the Petersen graph [21], the Pappus graph [25], the octahedron graph [14], and the Heawood graph [19] - has resulted in the classification of connected elementary abelian regular covers admitting various types of subgroups of automorphisms. A similar approach, also based on linear criteria for lifting automorphisms, was proposed in [3], and has been used in order to find connected regular coverings with cyclic or elementary abelian group of covering transformation for the complete graph $K_{4}$ [6], the 3-dimensional cube graph $Q_{3}$ [7], the complete bipartite graph $K_{3,3}$ [4], and the Petersen graph [5].

Assuming that a group $G$ of automorphisms of $X$ lifts along a regular covering projection $\wp: \tilde{X} \rightarrow X$, the lifted group $\tilde{G}$ is an extension of the group of covering transformations $\mathrm{CT}(\wp)$ by $G$. Specific types of extensions have usually a strong impact on structural properties of the covering graph $\tilde{X}$. In this context, the following two cases deserve special attention: (i) $\tilde{G}$ is a split extension of $\mathrm{CT}(\wp)$ by $G$, and in particular, (ii) $\tilde{G}$ is a direct split extension of $\mathrm{CT}(\wp)$ by $G$. For short we say that $G$ lifts as a split extension or as a direct split extension, respectively. In the former case there exists, by definition, a complement $\bar{G}$ of $\operatorname{CT}(\wp)$ within $\tilde{G}$, and a normal complement $\bar{G}$ of $\mathrm{CT}(\wp)$ in the latter. This allows us to compare actions of two isomorphic groups, $G$ on $X$ and $\bar{G}$ on $\tilde{X}$, where $\bar{G}$ projects isomorphically onto $G$ along $\wp$. However, it can happen that the complement is not unique, and what is more, different complements can exhibit different actions on $\tilde{X}$. Therefore, the analysis can be quite complicated. Certain algorithmic aspects related to the question of how difficult is to test conditions (i) and (ii) are considered in [22].

According to particular kinds of actions that can arise from complements, two extremal cases seem to stand out: (iii) there exists a complement $\bar{G}$ that acts transitively on the covering graph $\tilde{X}$, and (iv), there exists a complement $\bar{G}$ that is sectional. By this we mean that there is a section of $\tilde{X}-$ a set of vertices containing at most one vertex from each fibre - invariant under the action of $\bar{G}$. For short we say that $G$ lifts as a transitive split extension or as a sectional split extension, respectively. Clearly, one might further restrict conditions (iii) and (iv) to normal complements. Certain particular questions along these lines have been addressed in [1, 8, 16, 17].

Motivated by the above discussion, the following problem is of interest. Given a connected graph $X$ and a group $G$ of its automorphisms, find all connected regular covering projections $\wp: \tilde{X} \rightarrow X$ along which $G$ lifts in a prescribed way. In this paper we restrict to case (iv) - we introduce a method for finding regular coverings along which $G$ lifts as a sectional split extension.

The basic idea behind our approach is the following. First, we take the cone $\widehat{X}$ over 
the graph $X$ obtained by adding a new vertex $*$ joined to every vertex of $X$, together with the group of automorphisms $\widehat{G}$ of $\widehat{X}$ that fixes $*$ and acts on $X$ as the group $G$. Next, the condition for lifting $G$ as a sectional split extension is reduced to the general lifting problem of finding regular coverings of $\widehat{X}$ admitting the lift of $\widehat{G}$. Consequently, the original problem can be solved as soon as the general lifting problem can be solved. Our approach is illustrated on a concrete example: we construct all connected elementary abelian regular coverings of the complete graph $K_{4}$ along which a cyclic group of order 4 lifts as a sectional split extension.

The rest of the paper is organized as follows. In Section 2 we review some preliminary concepts about regular graph covers and lifting automorphisms. In Section 3 we devise a method for constructing connected regular covering projections along which $G$ lifts as a sectional split extension. A detailed example is provided in Section 4.

\section{Preliminaries}

A graph is an ordered quadruple $X=\left(D, V\right.$; beg, $\left.{ }^{-1}\right)$, where $D_{X}=D$ and $V_{X}=V$ are disjoint sets of darts and vertices, respectively, beg is a mapping that assigns to each dart $x$ its initial vertex $\operatorname{beg}(x)$, and ${ }^{-1}$ is an involution interchanging every dart $x$ and its inverse dart $x^{-1}$. For a dart $x$, its terminal vertex is the vertex end $(x)=\operatorname{beg}\left(x^{-1}\right)$. The orbits of ${ }^{-1}$ are called edges. An edge $e=\left\{x, x^{-1}\right\}$ is called a link whenever $\operatorname{beg}(x) \neq \operatorname{end}(x)$. If $\operatorname{beg}(x)=\operatorname{end}(x)$, then the respective edge is either a loop or a semi-edge, depending on whether $x \neq x^{-1}$ or $x=x^{-1}$, respectively. All graphs in this paper are assumed to be finite, meaning that the sets of vertices and darts are finite.

A graph homomorphism $f: Y \rightarrow X$ is an adjacency preserving mapping taking darts to darts and vertices to vertices, or more precisely, $f(\operatorname{beg}(x))=\operatorname{beg}(f(x))$ and $f\left(x^{-1}\right)=$ $f(x)^{-1}$. An isomorphism is a bijective homomorphism. An isomorphism of a graph onto itself is an automorphism. All automorphisms of a graph $X$ together with composition of automorphisms constitute the automorphism group Aut $(X)$.

A surjective homomorphism $\wp: \tilde{X} \rightarrow X$ is called a regular covering projection if there exists a semi-regular subgroup $S_{\wp}$ of $\operatorname{Aut}(\tilde{X})$ such that its vertex orbits and dart orbits coincide with vertex fibres $\wp^{-1}(v), v \in V_{X}$, and dart fibres $\wp^{-1}(x), x \in D_{X}$, respectively. Two regular covering projections $\wp: \tilde{X} \rightarrow X$ and $\wp^{\prime}: \tilde{X}^{\prime} \rightarrow X$ are isomorphic if there exist an automorphism $g$ of $X$ and an isomorphism $\tilde{g}: \tilde{X} \rightarrow \tilde{X}^{\prime}$ such that the following diagram

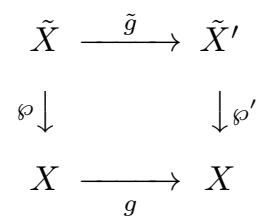

commutes. In particular, if $g=$ id then $\wp$ and $\wp^{\prime}$ are equivalent. If, in the above setting, $\tilde{X}=\tilde{X}^{\prime}$ and $\wp=\wp^{\prime}$, then we say that $g$ lifts along $\wp$ or that $\tilde{g}$ is a lift of $g$ along $\wp$. A group $G \leq \operatorname{Aut}(X)$ lifts if all $g \in G$ lift. The collection of all lifts of all elements in $G$ forms a subgroup $\tilde{G} \leq \operatorname{Aut}(\tilde{X})$, the lift of $G$. In particular, the lift of the trivial group is known as the group of covering transformations and denoted by $\mathrm{CT}(\wp)$. Observe that $\tilde{G}$ is an extension of $\mathrm{CT}(\wp)$ by $G$. Furthermore, if $G$ lifts along a given projection $\wp$, then it lifts along any covering projection equivalent to $\wp$. This allows us to study lifts of automorphisms combinatorially in terms of voltage assignments, a concept that we are 
going to describe now.

Let $X$ be a graph and let $N$ be an (abstract) group, called the voltage group. Assign to each dart $x$ of $X$ a voltage $\zeta_{x} \in N$ in such a way that $\zeta_{x^{-1}}=\zeta_{x}^{-1}$. Such a function $\zeta: D_{X} \rightarrow N$ is called a voltage assignment on $X$. Further, construct the derived graph $\operatorname{Cov}(\zeta)$ with vertex set $V_{X} \times N$ and dart set $D_{X} \times N$, where $\operatorname{beg}(x, n)=(\operatorname{beg}(x), n)$ and $(x, n)^{-1}=\left(x^{-1}, n \zeta_{x}\right)$. The projection onto the first coordinate $\wp_{\zeta}: \operatorname{Cov}(\zeta) \rightarrow X$ is then the derived regular covering projection, where the required semi-regular subgroup $S_{\wp_{\zeta}}$ of $\operatorname{Aut}(\operatorname{Cov}(\zeta))$ arises from the action of $N$ on the second coordinate by left multiplication on itself. Conversely, any regular covering projection $\wp: \tilde{X} \rightarrow X$ can be reconstructed by a voltage assignment $\zeta$ on $X$ such that the projection $\wp_{\zeta}$ derived from $\zeta$ is equivalent to $\wp$. Moreover, one can assume that the voltage assignment $\zeta$ is $\mathcal{T}$-reduced for some arbitrarily chosen spanning tree $\mathcal{T}$ of $X$, meaning that $\zeta_{x}=1$ for all darts $x$ in $\mathcal{T}$, see [9] for more details.

Consider now a regular covering projection $\wp$ of connected graphs. Then we say that $\wp$ is connected. Further, the semi-regular group $S_{\wp}$ is equal to CT $(\wp)$, and the voltage assignment $\zeta$ that reconstructs the projection $\wp$ is valued in the voltage group $N \cong \mathrm{CT}(\wp)$ (viewed as an abstract group). Such a voltage assignment $\zeta$ is also called connected. It is well known that $\zeta$ is connected if and only if each element of $N$ appears as the voltage of some closed walk. Furthermore, by the basic lifting lemma $[15,16]$, an automorphism $g$ of $X$ lifts along $\wp_{\zeta}$ if and only if each closed walk with trivial voltage is mapped by $g$ to a walk with trivial voltage.

Two assignments $\zeta$ and $\zeta^{\prime}$ on $X$ are equivalent whenever the respective derived regular covering projections $\wp_{\zeta}$ and $\wp_{\zeta^{\prime}}$ are equivalent. Assuming that both assignments are connected and valued in $N$, then they are equivalent if and only if there exists an automorphism of $N$ mapping $\zeta_{W}$ to $\zeta_{W}^{\prime}$ for each closed walk $W$ at $u_{0}$ [27].

For a given connected graph $X$ and subgroup $G \leq \operatorname{Aut}(X)$, the problem of finding regular covering projections $\wp$ along which $G$ lifts is very difficult in general. However, in the case of elementary abelian regular coverings $\wp-$ that is, when $\mathrm{CT}(\wp)$ is isomorphic to an elementary abelian group - the necessary and sufficient lifting condition can be stated combinatorially by means of voltages as follows, see [19].

Let $p$ be a prime. The first homology group $H_{1}\left(X ; \mathbb{Z}_{p}\right)$ is generated by the (directed) cycles of $X$ and is isomorphic to the elementary abelian group $\mathbb{Z}_{p}^{r}$, where $r$ is the Betti number of the graph $X$. The group $H_{1}\left(X ; \mathbb{Z}_{p}\right)$ is usually viewed as a vector space over $\mathbb{Z}_{p}$ of dimension $r$. Since each automorphism $\alpha \in \operatorname{Aut}(X)$ maps a cycle in $X$ to a cycle in $X$, there is a natural action of $\alpha$ on $H_{1}\left(X ; \mathbb{Z}_{p}\right)$ which induces a linear transformation $\alpha^{\#}$ of $H_{1}\left(X ; \mathbb{Z}_{p}\right)$. Choose a spanning tree $\mathcal{T}$ of $X$ and exactly one dart from each edge $\left\{x, x^{-1}\right\}$ that is not contained in $\mathcal{T}$. Then the sequence $x_{1}, x_{2}, \ldots, x_{r} \in D_{X} \backslash D_{\mathcal{T}}$ of all such darts naturally defines an (ordered) basis $\mathcal{B}_{\mathcal{T}}=\left\{C_{1}, C_{2}, \ldots, C_{r}\right\}$ of $H_{1}\left(X ; \mathbb{Z}_{p}\right)$, where $C_{i}$ is the cycle arising from the spanning tree $\mathcal{T}$ and the dart $x_{i}$. Next, denote the matrix representation of $\alpha^{\#}$ with respect to the basis $\mathcal{B}_{\mathcal{T}}$ by $M_{\alpha} \in \mathbb{Z}_{p}^{r, r}$. Thus, a subgroup $G \leq \operatorname{Aut}(X)$ induces a subgroup $M_{G}=\left\{M_{g} \mid g \in G\right\} \leq \mathrm{GL}\left(r, \mathbb{Z}_{p}\right)$. By $M_{G}^{t}$ we denote the dual group consisting of all transposes of matrices in $M_{G}$.

Theorem 2.1. ([19, Proposition 6.3, Corollary 6.5]) With the notation above, let $\zeta: D_{X} \rightarrow$ $\mathbb{Z}_{p}^{d, 1}$ be a $\mathcal{T}$-reduced voltage assignment on $X$, and let $Z \in \mathbb{Z}_{p}^{d, r}$ be the matrix with columns

$$
\zeta_{x_{1}}, \zeta_{x_{2}}, \ldots, \zeta_{x_{r}}
$$

If $Z$ has rank $d$, then the derived graph $\operatorname{Cov}(\zeta)$ is connected and the following hold: 
(i) A group $G \leq \operatorname{Aut}(X)$ lifts along $\wp_{\zeta}: \operatorname{Cov}(\zeta) \rightarrow X$ if and only if the columns of $Z^{t}$ form a basis of a $M_{G}^{t}$-invariant d-dimensional subspace $\mathcal{S}(\zeta)$ of $\mathbb{Z}_{p}^{r, 1} \cong H_{1}\left(X ; \mathbb{Z}_{p}\right)$.

(ii) If $\zeta^{\prime}: D_{X} \rightarrow \mathbb{Z}_{p}^{d, 1}$ is another voltage assignment on $X$ satisfying the above conditions, then $\wp_{\zeta^{\prime}}$ is equivalent to $\wp_{\zeta}$ if and only if $\mathcal{S}\left(\zeta^{\prime}\right)=\mathcal{S}(\zeta)$. Moreover, $\wp_{\zeta^{\prime}}$ is isomorphic to $\wp_{\zeta}$ if and only if there exists an automorphism $\alpha \in \operatorname{Aut}(X)$ such that the matrix $M_{\alpha}^{t}$ maps $\mathcal{S}\left(\zeta^{\prime}\right)$ onto $\mathcal{S}(\zeta)$.

By Theorem 2.1, we can find all pairwise nonequivalent connected elementary abelian regular coverings of $X$ along which $G$ lifts - in terms of voltages - as follows. First find a basis $\left\{u_{1}, u_{2}, \ldots, u_{d}\right\}$ for each $M_{G}^{t}$-invariant subspace $U$ of $\mathbb{Z}_{p}^{r, 1}$. Next, for each basis $\left\{u_{1}, u_{2}, \ldots, u_{d}\right\}$ consider a matrix $Z$ with rows $u_{1}^{t}, u_{2}^{t}, \ldots, u_{d}^{t}$, and then define the voltage assignment $\zeta^{U}: D_{X} \rightarrow \mathbb{Z}_{p}^{d, 1}$, mapping dart $x_{i}$ to the $i$-th column of $Z, i=1,2, \ldots, r$, and mapping all darts of $\mathcal{T}$ to the trivial voltage. Observe that the choice of a spanning tree together with a sequence $x_{1}, x_{2}, \ldots, x_{r}$ as well as choosing a basis for an invariant subspace is irrelevant as long as we consider regular coverings up to equivalence. Thus, the problem of finding connected elementary abelian regular coverings along which a given group of automorphisms lifts translates to a purely algebraic question of finding invariant subspaces of finite linear groups.

In this context, let $A \in \mathbb{Z}_{p}^{n, n}$ be an $n \times n$ matrix over a field $\mathbb{Z}_{p}$, acting as a linear transformation on the column vector space $\mathbb{Z}_{p}^{n, 1}$. Next, let $\kappa_{A}(x)=f_{1}(x)^{n_{1}} f_{2}(x)^{n_{2}} \cdots f_{k}(x)^{n_{k}}$ be the characteristic polynomial and $m_{A}(x)=f_{1}(x)^{s_{1}} f_{2}(x)^{s_{2}} \cdots f_{k}(x)^{s_{k}}$ the minimal polynomial of $A$ where polynomials $f_{i}$ are pairwise distinct and irreducible over $\mathbb{Z}_{p}$. Then $\mathbb{Z}_{p}^{n, 1}$ can be written as a direct sum of the $A$-invariant subspaces

$$
\mathbb{F}^{n, 1}=\operatorname{Ker} f_{1}(A)^{s_{1}} \oplus \operatorname{Ker} f_{2}(A)^{s_{2}} \oplus \cdots \oplus \operatorname{Ker} f_{k}(A)^{s_{k}} .
$$

Moreover, all $A$-invariant subspaces appear as direct sums of some $A$-invariant subspaces of $\operatorname{Ker} f_{i}(A)^{s_{i}}$.

As for finding common invariant subspaces of a finite linear group, we can often exploit Maschke's theorem which states that if the characteristic of the field does not divide the order of the group, then the representation is completely reducible. In this case one essentially needs to find just the minimal common invariant subspaces. In particular, if the order of the matrix $A$ is not divisible by $p$, each $A$-invariant subspace of $\mathbb{Z}_{p}^{n, 1}$ is a direct sum of the minimal ones. For a more detailed description of finding invariant subspaces we refer the reader to [10].

\section{Sectional split extensions}

We start by giving a more precise definition of a sectional split extension mentioned in the Introduction. Let $\wp: \tilde{X} \rightarrow X$ be a regular covering projection of connected graphs, and let $\Omega$ be a nonempty set of vertices of $X$. A section over $\Omega$ is a set of vertices $\bar{\Omega}$ of $\tilde{X}$ containing exactly one vertex from each vertex fibre over $\Omega$. Further, let $G$ be a group of automorphisms of $X$. Assuming that $\Omega$ is invariant under the action of $G$, we say that $G$ lifts along $\wp$ to $\tilde{G}$ as a sectional split extension over $\Omega$ if the following two conditions are met: (a) $G$ lifts along $\wp$ and (b) there exist a complement $\bar{G}$ to $\operatorname{CT}(\wp)$ within $\tilde{G}$ and a section $\bar{\Omega}$ over $\Omega$ that is invariant under the action of $\bar{G}$. Such a complement is called sectional over $\Omega$. The necessary and sufficient conditions for $G$ to lift as a sectional split extension over $\Omega$ in terms of voltages were given by Malnič et al. This is summarized in the following theorem. 
Theorem 3.1. ([16, Theorem 9.1, Theorem 9.3]) With the notation and assumptions above, a group $G$ lifts along $\wp$ as a sectional split extension over $\Omega$ if and only if $\wp$ can be reconstructed by a voltage assignment $\zeta$ on $X$ such that the following condition

$$
\zeta_{W}=1 \Rightarrow \zeta_{g W}=1
$$

holds for each automorphism $g \in G$ and each walk $W$ in $X$ with both its endpoints in $\Omega$.

Firstly, note that this theorem is an extended version of an old result of Biggs [1], retold in a different language. Secondly, Malnič took this result further in [17], and used it to sketch a method for testing whether $G$ lifts along $\wp$ as a sectional split extension over $\Omega$. The approach is based on introducing a new vertex joined to every vertex of $\Omega$, and then converting condition (3.1) to the general lifting problem (but no proof is given). In order to exploit this idea in another direction (see below), we introduce the following notation.

The cone $\widehat{X}(\Omega)$ over the graph $X$ is the graph obtained by adding a new vertex $*$ joined to every vertex of $\Omega$. Assuming that $\Omega$ is invariant under the action of $G$, we denote by $\widehat{G}$ the group of automorphisms of $\widehat{X}(\Omega)$ that fixes $*$ and acts on $X$ as the group $G$. Also, for any voltage assignment $\zeta$ on $X$, we extend $\zeta$ to a voltage assignment $\widehat{\zeta}$ on $\widehat{X}(\Omega)$ by assigning the trivial voltage to the extra darts. More precisely,

$$
\widehat{\zeta}_{x}= \begin{cases}\zeta_{x}, & x \in D_{X} ; \\ 1, & x \in D_{\widehat{X}(\Omega)} \backslash D_{X} .\end{cases}
$$

Conversely, for a voltage assignment $\zeta$ on $\widehat{X}(\Omega)$ being trivial on the set of extra darts we denote by $\bar{\zeta}$ the restriction of $\zeta$ to $X$. Clearly, if $\zeta$ is not trivial on the set of extra darts, then we can always find an equivalent assignment that is. For example, we may choose a spanning tree $\mathcal{T}^{*}$ of $\widehat{X}(\Omega)$ such that all extra darts are included in $\mathcal{T}^{*}$, and then take an equivalent $\mathcal{T}^{*}$-reduced voltage assignment. Moreover, the following holds.

Proposition 3.2. Let $\zeta$ and $\zeta^{\prime}$ be two equivalent connected voltage assignments on $\widehat{X}(\Omega)$, that are trivial on the set of extra darts $D_{\widehat{X}(\Omega)} \backslash D_{X}$. Then their restrictions $\bar{\zeta}$ and $\overline{\zeta^{\prime}}$ to $X$ are also equivalent. Hence they are either both connected or both disconnected.

Proof. By definition of equivalence, there exists an isomorphism $\tilde{g}$ from the derived graph $\operatorname{Cov}(\zeta)$ to the derived graph $\operatorname{Cov}\left(\zeta^{\prime}\right)$ such that $\wp_{\zeta}=\tilde{g} \wp_{\zeta^{\prime}}$. Clearly, $\tilde{g}$ maps the vertex fibre $\wp_{\zeta}^{-1}(*)$ to the vertex fibre $\wp_{\zeta^{\prime}}^{-1}(*)$. Therefore, when restricting to $X$, the isomorphism $\tilde{g}$ induces an isomorphism from the derived graph $\operatorname{Cov}(\bar{\zeta})$ to $\operatorname{Cov}\left(\bar{\zeta}^{\prime}\right)$ that gives rise to an equivalence of $\bar{\zeta}$ and $\bar{\zeta}^{\prime}$. It is then obvious that isomorphic graphs are either both connected or both disconnected, as required.

We are now ready to forge a link between connected regular coverings of $X$ along which $G$ lifts as a sectional split extension over $\Omega$, and connected regular coverings of $\widehat{X}(\Omega)$ admitting the lift of $\widehat{G}$. For completeness, we explicitly record the following theorem and provide the proof.

Theorem 3.3. Let $\wp: \tilde{X} \rightarrow X$ be a regular covering projection of connected graphs, and let $G$ be a group of automorphisms of $X$. Suppose that a nonempty subset $\Omega$ of vertices of $X$ is invariant under the action of $G$. Then the group $G$ lifts along $\wp$ as a sectional split extension over $\Omega$ if and only if $\wp$ can be reconstructed by a voltage assignment $\zeta$ on $X$ such that $\widehat{G}$ lifts along the derived regular covering projection $\wp_{\widehat{\zeta}}: \operatorname{Cov}(\widehat{\zeta}) \rightarrow \widehat{X}(\Omega)$. 
Proof. Suppose that $G$ lifts along $\wp$ as a sectional split extension over $\Omega$. By Theorem 3.1, there exists a voltage assignment $\zeta$ on $X$ that reconstructs $\wp$ and satisfies condition (3.1). Extend $\zeta$ to a voltage assignment $\widehat{\zeta}$. We will show that $\widehat{G}$ lifts along the projection $\wp_{\widehat{\zeta}}$ derived from $\widehat{\zeta}$. Let $W^{*}$ be a closed walk at $*$ in $\widehat{X}(\Omega)$ with $\widehat{\zeta}_{W^{*}}=1$, and let $g^{*} \in \widehat{G}$. In view of the basic lifting lemma we need to show that $\widehat{\zeta}_{g^{*} W^{*}}=1$. Write $W^{*}$ as a concatenation $W^{*}=W_{1}^{*} W_{2}^{*} \ldots W_{k}^{*}$ of closed walks at $*$ such that $W_{i}^{*}=P_{i} W_{i} Q_{i}^{-1}$, where $W_{i}: u_{i} \rightarrow v_{i}$ is a walk in $X$ with both its endpoints $u_{i}$ and $v_{i}$ in $\Omega$, while $P_{i}: * \rightarrow u_{i}$ and $Q_{i}: * \rightarrow v_{i}$ are walks of length 1 , for $i=1,2, \ldots, k$. Observe that $\zeta_{W_{1}} \zeta_{W_{2}} \ldots \zeta_{W_{k}}=$ 1. Now choose a vertex $u_{0} \in \Omega$. Let $R_{i}: u_{0} \rightarrow u_{i}$ and $S_{i}: u_{0} \rightarrow v_{i}$ be walks with $\zeta_{R_{i}}=\zeta_{S_{i}}=1$, for $i=1,2, \ldots, k$ (note that such walks always exist). Then the product of walks $W=\prod_{i=1}^{k} R_{i} W_{i} S_{i}^{-1}$ is a closed walk at $u_{0}$ with $\zeta_{W}=\zeta_{W_{1}} \zeta_{W_{2}} \ldots \zeta_{W_{k}}=1$. By condition (3.1) we have that $\zeta_{g W}=1$ as well as $\zeta_{g R_{j}}=\zeta_{g S_{j}}=1$, for $i=1,2, \ldots, k$. Thus $\zeta_{g W_{1}} \zeta_{g W_{2}} \ldots \zeta_{g W_{k}}=1$ implies that $\widehat{\zeta}_{g^{*} W^{*}}=1$, as required.

Conversely, suppose that $\wp$ is reconstructed by a voltage assignment $\zeta$ on $X$ such that $\widehat{G}$ lifts along the covering projection $\wp_{\widehat{\zeta}}$. By Theorem 3.1, it is sufficient to prove that $\zeta$ satisfies condition (3.1). Consider a walk $W: u \rightarrow v$ in $X$ with both its endpoints $u$ and $v$ in $\Omega$ such that $\zeta_{W}=1$. Let $P: * \rightarrow u$ and $Q: * \rightarrow v$ be the (unique) walks of length 1 in $\widehat{X}(\Omega)$. Then the closed walk $W^{*}=P W Q^{-1}$ at $*$ has voltage $\widehat{\zeta}_{W^{*}}=1$. By the basic lifting lemma we have $\widehat{\zeta}_{g^{*} W^{*}}=1$ for any automorphism $g^{*} \in \widehat{G}$. Hence $\zeta_{g W}=1$, completing the proof.

Coming back to methods for testing whether $G$ lifts along $\wp$ as a sectional split extension over $\Omega$, one possibility would be to use the latter theorem. However, from computational point of view that would be inefficient, since one has to seek for an appropriate voltage assignment that reconstructs the cover. For a more adequate approach to this problem we refer the reader to [23].

As already mentioned, Theorem 3.3 can be efficiently exploited in another direction: given a connected graph $X$, a group $G$ of its automorphisms, and a nonempty subset $\Omega \subseteq V_{X}$ invariant under the action of $G$, find, up to equivalence, all connected regular coverings $\wp: \tilde{X} \rightarrow X$ along which $G$ lifts as a sectional split extension over $\Omega$. As a first step towards this aim we need to find, in view of Proposition 3.1 and Theorem 3.3, all pairwise nonequivalent connected regular coverings of $\widehat{X}(\Omega)$ along which the group $\widehat{G}$ lifts - combinatorially reconstructed in terms of voltage assignments $\zeta$ being trivial on the set of extra darts. Although each $\zeta$ is connected - as it reconstructs a connected cover - its restriction $\bar{\zeta}$ to $X$, however, might be disconnected. Thus, additional testing whether $\bar{\zeta}$ is connected is required. These remarks are formally gathered in the following theorem.

Theorem 3.4. Let $X$ be a connected graph and $\Omega$ a nonempty subset of vertices of $X$ that is invariant under the action of a group of automorphisms $G \leq \operatorname{Aut}(X)$. Further, let $\zeta$ be a voltage assignment on $\widehat{X}(\Omega)$ that is trivial on the set of extra darts $D_{\widehat{X}(\Omega)} \backslash D_{X}$ and gives rise to a connected regular covering projection along which the group $\widehat{G}$ lifts. If the restriction $\bar{\zeta}$ to $X$ is connected, then $G$ lifts along the derived regular covering projection $\wp_{\bar{\zeta}}$ as a sectional split extension over $\Omega$. Moreover, any connected regular covering of $X$ along which $G$ lifts as a sectional split extension over $\Omega$ arises in this way.

Remark 3.5. Even if $\zeta$ and $\zeta^{\prime}$ are two nonequivalent connected assignments on $\widehat{X}(\Omega)$ such that their restrictions $\bar{\zeta}$ and $\bar{\zeta}^{\prime}$ to $X$ are connected, it still might happened that $\bar{\zeta}$ and $\bar{\zeta}^{\prime}$ are 
equivalent. Thus, additional testing is needed.

Now we can more precisely summarize our approach. First, construct all voltage assignments $\zeta$ on $\widehat{X}(\Omega)$ giving rise to pairwise nonequivalent connected regular covering projections along which $\widehat{G}$ lifts. Next, consider their restrictions $\bar{\zeta}$ to $X$ and remove the disconnected ones. Finally, do further reduction to obtain all voltage assignments on $X$ giving rise to pairwise nonequivalent connected regular covering projections along which $G$ lifts as a sectional split extension over $\Omega$.

\section{Elementary abelian regular covers of $K_{4}$}

In light of the discussion in Section 3 we now give an example to illustrate our approach. Let $X=K_{4}$ be the complete graph on the vertex set $V_{X}=\{1,2,3,4\}$, and let $\Omega=V_{X}$. Further, denote by $g=(1234) \in \operatorname{Aut}(X)$ the automorphism of $X$. We compute all voltage assignments on $X$ giving rise to pairwise nonequivalent connected elementary abelian regular covering projections along which the cyclic group $G=\langle g\rangle$ lifts as a sectional split extension over $\Omega$.

To start with, we need to find all voltage assignments on $\widehat{X}(\Omega)$ giving rise to pairwise nonequivalent connected elementary abelian regular coverings along which the group $\widehat{G}=$ $\left\langle g^{*}\right\rangle$ lifts. Let $\mathcal{T}^{*}$ be the spanning tree of $\widehat{X}(\Omega)$ consisting of all extra darts, and let

$$
x_{1}=(1,2), x_{2}=(2,3), x_{3}=(3,4), x_{4}=(4,1), x_{5}=(2,4), x_{6}=(3,1)
$$

denote the six cotree darts of $\widehat{X}(\Omega)$. Denote by $\mathcal{B}_{\mathcal{T}^{*}}=\left\{\vec{x}_{i} \mid 1 \leq i \leq 6\right\}$ the ordered basis of the vector space $H_{1}\left(\widehat{X}(\Omega) ; \mathbb{Z}_{p}\right)$, where $\vec{x}_{i}$ is the cycle arising from the spanning tree $\mathcal{T}^{*}$ and the dart $x_{i}$. Next, in view of the remarks given in Preliminaries, let $\left(g^{*}\right)^{\#}$ be the linear transformation of $H_{1}\left(\widehat{X}(\Omega) ; \mathbb{Z}_{p}\right)$ induced by the natural action of $g^{*}$ on $H_{1}\left(\widehat{X}(\Omega) ; \mathbb{Z}_{p}\right)$, and let $M_{g^{*}} \in \mathbb{Z}_{p}^{6,6}$ be its matrix representation with respect to the basis $\mathcal{B}_{\mathcal{T}^{*}}$. By computation we obtain that

$$
A=M_{g^{*}}^{t}=\left[\begin{array}{cccccc}
0 & 1 & 0 & 0 & 0 & 0 \\
0 & 0 & 1 & 0 & 0 & 0 \\
0 & 0 & 0 & 1 & 0 & 0 \\
1 & 0 & 0 & 0 & 0 & 0 \\
0 & 0 & 0 & 0 & 0 & 1 \\
0 & 0 & 0 & 0 & -1 & 0
\end{array}\right]
$$

By Theorem 2.1, we need to find $A$-invariant subspaces of $\mathbb{Z}_{p}^{6,1}$. However, note that every elementary abelian regular $\mathbb{Z}_{p}^{d, 1}$-cover of $X$ is disconnected if the dimension $d$ is higher that the Betti number of $X$. Since the Betti number of $X$ is three, it is therefore enough to find all $A$-invariant subspaces of dimension at most three. These subspaces define $\mathcal{T}^{*}$-reduced voltage assignments

$$
\zeta: D_{\widehat{X}(\Omega)} \rightarrow \mathbb{Z}_{p}^{d, 1}, \quad d=1,2,3
$$

on $\widehat{X}(\Omega)$ that give rise to pairwise nonequivalent connected regular coverings of $\widehat{X}(\Omega)$ along which $\widehat{G}$ lifts. In addition, as already explained in the previous section, their restrictions $\bar{\zeta}$ to $X$ might still be disconnected as well as connected but equivalent.

In order to test whether the restriction $\bar{\zeta}$ to $X$ stays connected, let $\mathcal{T}$ be the spanning tree of $X$ consisting of the edges $\{1,2\},\{1,3\}$ and $\{1,4\}$. Denote by $C_{1}, C_{2}$ and $C_{3}$ cycles 
arising from the spanning tree $\mathcal{T}$ and darts $x_{2}, x_{3}$ and $x_{5}$, respectively. The connectedness condition, relative to the ordered basis $\mathcal{B}_{\mathcal{T}}=\left\{C_{1}, C_{2}, C_{3}\right\}$ of $H_{1}\left(X ; \mathbb{Z}_{p}\right)$, translates to the requirement that the voltages

$$
\begin{aligned}
& \bar{\zeta}_{C_{1}}=\zeta_{C_{1}}=\zeta_{x_{1}}+\zeta_{x_{2}}+\zeta_{x_{6}}, \\
& \bar{\zeta}_{C_{2}}=\zeta_{C_{2}}=\zeta_{x_{3}}+\zeta_{x_{4}}-\zeta_{x_{6}}, \\
& \bar{\zeta}_{C_{3}}=\zeta_{C_{3}}=\zeta_{x_{1}}+\zeta_{x_{4}}+\zeta_{x_{5}}
\end{aligned}
$$

generate the voltage group $\mathbb{Z}_{p}^{d, 1}$. As for the test of equivalence, let $\zeta$ and $\zeta^{\prime}$ be two $\mathcal{T}^{*}$ reduced voltage assignments on $\widehat{X}(\Omega)$ arising from two different $d$-dimensional subspaces $U$ and $U^{\prime}$ of $\mathbb{Z}_{p}^{6,1}$, respectively. Suppose that their restrictions $\bar{\zeta}$ and $\overline{\zeta^{\prime}}$ are connected. Then $\bar{\zeta}$ and $\bar{\zeta}^{\prime}$ are equivalent, in view of [27], if and only if there exists an automorphism of $\mathbb{Z}_{p}^{d, 1}$ mapping

$$
\zeta_{C_{1}} \mapsto \zeta_{C_{1}}^{\prime}, \zeta_{C_{2}} \mapsto \zeta_{C_{2}}^{\prime}, \text { and } \zeta_{C_{3}} \mapsto \zeta_{C_{3}}^{\prime}
$$

For the purpose of finding $A$-invariant subspaces, note that $\kappa_{A}(x)=\left(x^{4}-1\right)\left(x^{2}+1\right)$ is the characteristic polynomial of $A$, while

$$
m_{A}(x)=x^{4}-1
$$

is its minimal polynomial. Further, observe that the factorization of $m_{A}(x)$ into irreducible factors over $\mathbb{Z}_{p}$ depends on the congruence class of $p$ modulo 4 , namely

$$
m_{A}(x)= \begin{cases}(x-1)(x+1)\left(x^{2}+1\right), & p \equiv 3(\bmod 4) \\ (x-1)(x+1)(x-i)(x+i), & p \equiv 1(\bmod 4), i^{2}=-1 \\ (x-1)^{4}, & p=2\end{cases}
$$

Therefore the analysis splits into three cases.

Case $p \equiv 3(\bmod 4)$.

In this case the representation of the group $\langle A\rangle$ is completely reducible, by Maschke's theorem. The eigenvalues are 1 and -1 , both of multiplicity 1 . The respective eigenspaces are $\mathcal{L}_{A}(1)=\left\langle v_{1}\right\rangle$ and $\mathcal{L}_{A}(-1)=\left\langle v_{2}\right\rangle$, where

$$
v_{1}=(1,1,1,1,0,0)^{t} \text { and } v_{2}=(1,-1,1,-1,0,0)^{t} .
$$

The whole space splits into a direct sum of $A$-invariant subspaces

$$
\mathbb{Z}_{p}^{6,1}=\mathcal{L}_{A}(1) \oplus \mathcal{L}_{A}(-1) \oplus \operatorname{Ker}\left(A^{2}+I\right)
$$

It is obvious that the 1 -dimensional $A$-invariant subspaces are $\mathcal{L}_{A}(1)$ and $\mathcal{L}_{A}(-1)$. The respective lists of voltages for the base homology cycles $C_{1}, C_{2}, C_{3}$ in $X$ are 2,2,2 for the one arising from $\mathcal{L}_{A}(1)$, and $0,0,0$ for the one arising from $\mathcal{L}_{A}(-1)$. Thus, only $\mathcal{L}_{A}(1)$ gives rise to a connected cover of $X$, while $\mathcal{L}_{A}(-1)$ does not.

Since the 2-dimensional $A$-invariant subspace arising from the direct sum $\mathcal{L}_{A}(1) \oplus$ $\mathcal{L}_{A}(-1)$ does not give a connected cover of $X$, all others are necessarily contained in $\operatorname{Ker}\left(A^{2}+I\right)$. These subspaces are of the form $\langle v, A v\rangle$, for $v \in \operatorname{Ker}\left(A^{2}+I\right)$. There are 
$p^{2}+1$ distinct subspaces. To check which of these give rise to connected covers of $X$, choose a basis of $\operatorname{Ker}\left(A^{2}+I\right)$, for instance

$$
\begin{aligned}
& b_{1}=(1,0,-1,0,0,0)^{t}, \\
& b_{2}=(0,1,0,-1,0,0)^{t}, \\
& b_{3}=(0,0,0,0,1,0)^{t}, \\
& b_{4}=(0,0,0,0,0,1)^{t} .
\end{aligned}
$$

An arbitrary vector $v \in \operatorname{Ker}\left(A^{2}+I\right)$ is then of the form $v=(a, b,-a,-b, c, d)^{t}$, for some $a, b, c, d \in \mathbb{Z}_{p}$, while $A v=(b,-a,-b, a, d,-c)^{t}$. For convenience we denote

$$
W_{a, b, c, d}=\left\langle(a, b,-a,-b, c, d)^{t},(b,-a,-b, a, d,-c)^{t}\right\rangle .
$$

Checking for connectedness gives that

$$
(a, b)^{t}+(b,-a)^{t}+(d,-c)^{t},(-a,-b)^{t}+(-b, a)^{t}-(d,-c)^{t} \text { and }(a, b)^{t}+(-b, a)^{t}+(c, d)^{t}
$$

should generate $\mathbb{Z}_{p}^{2,1}$. The condition is reduced to requiring that $(a+b+d,-a+b-c)^{t}$ and $(a-b+c, a+b+d)^{t}$ are linearly independent in $\mathbb{Z}_{p}^{2,1}$. Let $x=a+b+d$ and $y=a-b+c$. The vectors $(x, y)^{t}$ and $(-y, x)^{t}$ are linearly dependent if and only if $x^{2}+y^{2} \equiv 0(\bmod p)$ Since $p \equiv 3(\bmod 4)$, we must have $x \equiv 0(\bmod p)$ and $y \equiv 0(\bmod p)$. Thus a disconnected cover of $X$ is obtained if and only if $c=-a+b$ and $d=-a-b$; in this case $W_{a, b, c, d}$ is generated by

$$
v_{a, b}=a(1,0,-1,0,-1,-1)^{t}+b(0,-1,0,1,-1,1)^{t} \text { and } A v_{a, b} .
$$

Observe that any $v_{a, b}$ is contained in $\left\langle v_{1,0}, A v_{1,0}\right\rangle$. Hence $\left\langle v_{a, b}, A v_{a, b}\right\rangle=\left\langle v_{1,0}, A v_{1,0}\right\rangle$ for all $a, b \in \mathbb{Z}_{p}$. This is therefore the only $A$-invariant 2 -dimensional subspace giving rise to a disconnected cover of $X$. As for the remaining subspaces, these are $W_{a, b, c, d}$ where $(c, d) \neq(-a+b,-a-b)$. Furthermore, these subspaces all give rise to equivalent coverings of $X$. Indeed. Choose one of these subspaces, say

$$
W_{1,1,0,0}=\left\langle(1,1,-1,-1,0,0)^{t},(1,-1,-1,1,0,0)^{t}\right\rangle .
$$

Let $\zeta$ and $\zeta^{\prime}$ be two assignments arising from $W_{a, b, c, d}$ and $W_{1,1,0,0}$, respectively. The base homology cycles $C_{1}, C_{2}, C_{3}$ in $X$ have the following voltages

$$
\begin{array}{lll}
\zeta_{C_{1}}=(a+b+d,-a+b-c)^{t}, & \zeta_{C_{1}}^{\prime}=(2,0)^{t}, \\
\zeta_{C_{2}}=(-a-b-d, a-b+c)^{t}, & \zeta_{C_{2}}^{\prime}=(-2,0)^{t}, \\
\zeta_{C_{3}}=(a-b+c, a+b+d)^{t}, & \zeta_{C_{3}}^{\prime}=(0,2)^{t} .
\end{array}
$$

By computation one can check that there exists a matrix in $\operatorname{GL}\left(2, \mathbb{Z}_{p}\right) \cong \operatorname{Aut}\left(\mathbb{Z}_{p}^{2,1}\right)$ taking $\zeta_{C_{1}}, \zeta_{C_{2}}, \zeta_{C_{3}}$ to $\zeta_{C_{1}}^{\prime}, \zeta_{C_{2}}^{\prime}, \zeta_{C_{3}}^{\prime}$, respectively, if and only if $(c, d) \neq(-a+b,-a-b)$, and the claim is proved. As a representative of the above 2-dimensional subspaces we take $W_{1,1,0,0}$.

Any 3 -dimensional $A$-invariant subspace giving rise to a connected cover of $X$ is equivalent to the homological cover of $X$. So it is enough to find one such a subspace, if it exists. For instance, we may take the subspace $\mathcal{L}_{A}(1) \oplus W_{1,1,0,0}$, as the reader can easily check. 
Case $p \equiv 1(\bmod 4)$.

The representation of the group $\langle A\rangle$ is again completely reducible, by Maschke's theorem. The matrix $A$ is diagonalizable, having the diagonal form $\operatorname{diag}_{A}(1,-1, i, i,-i,-i)$.

Clearly, the 1-dimensional eigenspaces $\mathcal{L}_{A}(1)$ and $\mathcal{L}_{A}(-1)$ are the same as before, where only $\mathcal{L}_{A}(1)$ gives rise to a connected cover of $X$. As for the eigenvalues $i$ and $-i$ satisfying $i^{2} \equiv-1(\bmod p)$, the respective eigenspaces $\mathcal{L}_{A}(i)=\left\langle u_{i}, v_{i}\right\rangle$ and $\mathcal{L}_{A}(-i)=$ $\left\langle u_{-i}, v_{-i}\right\rangle$ are 2-dimensional, where

$$
\begin{array}{ll}
u_{i}=(1, i,-1,-i, 1, i)^{t}, & u_{-i}=(1,-i,-1, i, 1,-i)^{t}, \\
v_{i}=(1, i,-1,-i, 0,0)^{t}, & v_{-i}=(1,-i,-1, i, 0,0)^{t} .
\end{array}
$$

The 1-dimensional subspaces in $\mathcal{L}_{A}(i)$ can be conveniently parametrized as

$$
\begin{aligned}
W_{\infty}(i) & =\left\langle u_{i}\right\rangle, \\
W_{s}(i) & =\left\langle s u_{i}+v_{i}\right\rangle=\left\langle(s+1,(s+1) i,-(s+1),-(s+1) i, s, s i)^{t}\right\rangle, \quad s \in \mathbb{Z}_{p},
\end{aligned}
$$

while those in $\mathcal{L}_{A}(-i)$ can be parametrized as

$$
\begin{aligned}
W_{\infty}(-i) & =\left\langle u_{-i}\right\rangle, \\
W_{s}(-i) & =\left\langle s u_{-i}+v_{-i}\right\rangle=\left\langle(s+1,-(s+1) i,-(s+1),(s+1) i, s,-s i)^{t}\right\rangle, \quad s \in \mathbb{Z}_{p} .
\end{aligned}
$$

The conditions for connectedness of covers of $X$ arising from $W_{\infty}(i), W_{s}(i), W_{\infty}(-i)$ and $W_{s}(-i)$ become $i-2 \not \equiv 0(\bmod p), s(i-2) \not \equiv 1-i(\bmod p),-i-2 \not \equiv 0(\bmod p)$ and $s(-i-2) \not \equiv 1+i(\bmod p)$, respectively. We need to consider subcases $p \neq 5$ and $p=5$ separately.

Let $p \neq 5$. Then $i,-i \neq 2$, and there are $(2 p+1) 1$-dimensional subspaces giving rise to connected covers of $X$, namely the set

$$
\mathcal{W}_{i}=\left\{W_{s}(i) \mid s \in\left(\mathbb{Z}_{p} \backslash\left\{(1-i)(i-2)^{-1}\right\}\right) \cup\{\infty\}\right\}
$$

of $p$ subspaces in $\mathcal{L}_{A}(i)$, the set

$$
\mathcal{W}_{-i}=\left\{W_{s}(-i) \mid s \in\left(\mathbb{Z}_{p} \backslash\left\{(1+i)(-i-2)^{-1}\right\}\right) \cup\{\infty\}\right\}
$$

of $p$ subspaces in $\mathcal{L}_{A}(-i)$, and the subspace $\mathcal{L}_{A}(1)$. However, all subspaces in $\mathcal{W}_{i}$ give rise to equivalent coverings of $X$. To show this, let $\zeta$ and $\zeta^{\prime}$ be two assignments arising from $W_{s}(i)$ and $W_{\infty}(i)$, respectively. By computation we have

$$
\begin{array}{ll}
\zeta_{C_{1}}=(s+1)(1+i)+s i, & \zeta_{C_{1}}^{\prime}=1+2 i, \\
\zeta_{C_{2}}=-(s+1)(1+i)-s i=-\zeta_{C_{1}}, & \zeta_{C_{2}}^{\prime}=-1-2 i=-\zeta_{C_{1}}^{\prime}, \\
\zeta_{C_{3}}=(s+1)(1-i)+s=-i \zeta_{C_{1}}, & \zeta_{C_{3}}^{\prime}=2-i=-i \zeta_{C_{1}}^{\prime} .
\end{array}
$$

Clearly, there exists an automorphism of $\mathbb{Z}_{p}$ taking $\zeta_{C_{1}}, \zeta_{C_{2}}, \zeta_{C_{3}}$ to $\zeta_{C_{1}}^{\prime}, \zeta_{C_{2}}^{\prime}, \zeta_{C_{3}}^{\prime}$, respectively, if and only if $\zeta_{C_{1}} \neq 0$. In fact, we do have $\zeta_{C_{1}} \neq 0$ since $s \neq(1-i)(i-2)^{-1}$. Similarly, all subspaces in $\mathcal{W}_{-i}$ give rise to equivalent coverings of $X$. As a representative in $\mathcal{W}_{i}$ we choose $W_{0}(i)$, while in $\mathcal{W}_{-i}$ we choose $W_{0}(-i)$. In fact, there are exactly three pairwise nonequivalent connected coverings of $X$, namely the one arising from $\mathcal{L}_{A}(1)$, and 
the two coverings arising from $W_{0}(i)$ and $W_{0}(-i)$. The respective lists of voltages for the base homology cycles $C_{1}, C_{2}, C_{3}$ in $X$ are $2,2,2$ for the one arising from $\mathcal{L}_{A}(1)$, while $1+i,-1-i, 1-i$ and $1-i,-1+i, 1+i$ for the other two covers. The reader may check that there is no automorphism of $\mathbb{Z}_{p}$ taking any of these triples to any other.

Let $p=5$. Then for each $s \in \mathbb{Z}_{5}$ the subspace $W_{s}(2)$ gives rise to a connected cover of $X$, while the subspace $W_{\infty}(2)$ does not. On the other hand, for each $s \neq 3$ we obtain a connected cover of $X$ arising from $W_{s}(3)$, and one connected cover of $X$ arising from $W_{\infty}(3)$. Together with the cover of $X$ arising from $\mathcal{L}_{A}(1)$ we therefore have $2 p+1=11$ connected covers. If $\zeta$ denotes an assignment arising from $W_{s}(2)$, then the base homology cycles in $X$ have voltages $\zeta_{C_{1}}=3(s+1)+2 s=3, \zeta_{C_{2}}=-3(s+1)-2 s=-\zeta_{C_{1}}$, $\zeta_{C_{3}}=-(s+1)+s=-2 \zeta_{C_{1}}$. It is obvious that the subspaces $W_{s}(2), s \in \mathbb{Z}_{5}$, give rise to equivalent coverings of $X$. As a representative we take $W_{0}(2)$. Let now $\zeta$ be an assignment arising from $W_{s}(3)$, where $s \in \mathbb{Z}_{p}$ and $s \neq 3$. Further, let $\zeta^{\prime}$ denote an assignment arising from $W_{\infty}(3)$. Then we have

$$
\begin{array}{ll}
\zeta_{C_{1}}=4(s+1)+3 s=2 s-1, & \zeta_{C_{1}}^{\prime}=2, \\
\zeta_{C_{2}}=-4(s+1)-3 s=-\zeta_{C_{1}}, & \zeta_{C_{2}}^{\prime}=-2=-\zeta_{C_{1}}^{\prime}, \\
\zeta_{C_{3}}=-2(s+1)+s=-3 \zeta_{C_{1}}, & \zeta_{C_{3}}^{\prime}=-1=-3 \zeta_{C_{1}}^{\prime} .
\end{array}
$$

Clearly, multiplication by $s+2$ takes $\zeta_{C_{1}}, \zeta_{C_{2}}, \zeta_{C_{3}}$ to $\zeta_{C_{1}}^{\prime}, \zeta_{C_{2}}^{\prime}, \zeta_{C_{3}}^{\prime}$, respectively. As a representative we take $W_{0}(3)$. The reader may check that $\mathcal{L}_{A}(1), W_{0}(2)$ and $W_{0}(3)$ give rise to pairwise nonequivalent coverings of $X$.

Let us now consider the 2-dimensional subspaces. We shall need the following lemma.

Lemma 4.1. Let $\mathcal{T}^{*}$ be a spanning tree of $\widehat{X}(\Omega)$ such that all extra darts are included in $\mathcal{T}^{*}$, and let the sequence $x_{1}, x_{2}, \ldots, x_{n}$ contain exactly one dart from each edge not contained in $\mathcal{T}^{*}$. Further, let $U, U^{\prime}, W, W^{\prime}$ be subspaces of $\mathbb{Z}_{p}^{n, 1}$ such that $U \cap W=\{0\}=U^{\prime} \cap W^{\prime}$, and let $\zeta^{U}, \zeta^{U^{\prime}}, \zeta^{W}, \zeta^{W^{\prime}}, \zeta^{U \oplus W}, \zeta^{U^{\prime} \oplus W^{\prime}}$ denote $\mathcal{T}^{*}$-reduced voltage assignments on $\widehat{X}(\Omega)$, where the voltages of darts $x_{i}$ arise from $U, U^{\prime}, W, W^{\prime}, U \oplus W, U^{\prime} \oplus W^{\prime}$, respectively. Suppose that all their restrictions to $X$ are connected. If the restrictions of $\zeta^{U}$ and $\zeta^{U^{\prime}}$ are equivalent and the restrictions of $\zeta^{W}$ and $\zeta^{W^{\prime}}$ are equivalent, then the restrictions of $\zeta^{U \oplus W}$ and $\zeta^{U^{\prime} \oplus W^{\prime}}$ are also equivalent.

Proof. Since $U \cap W=\{0\}=U^{\prime} \cap W^{\prime}$ we may assume, up to equivalence of regular covering projections, that

$$
\zeta_{x}^{U \oplus W}=\left[\begin{array}{c}
\zeta_{x}^{U} \\
\zeta_{x}^{W}
\end{array}\right] \text { and } \zeta_{x}^{U^{\prime} \oplus W^{\prime}}=\left[\begin{array}{c}
\zeta_{x}^{U^{\prime}} \\
\zeta_{x}^{W^{\prime}}
\end{array}\right]
$$

for all darts $x$ in $\widehat{X}(\Omega)$. Let $r$ be the Betti number of $X$, and let $C_{1}, C_{2}, \ldots, C_{r}$ be an ordered basis of $H_{1}\left(X, \mathbb{Z}_{p}\right)$. Since the restrictions of $\zeta^{U}$ and $\zeta^{U^{\prime}}$ are equivalent, there exists an invertible matrix $A$ mapping voltages $\zeta_{C_{i}}^{U}$ to voltages $\zeta_{C_{i}}^{U^{\prime}}, i=1,2, \ldots, r$. Similarly, there exists an invertible matrix $B$ mapping voltages $\zeta_{C_{i}}^{W}$ to voltages $\zeta_{C_{i}}^{W^{\prime}}, i=1,2, \ldots, r$. Then the matrix

$$
\left[\begin{array}{ll}
A & \\
& B
\end{array}\right]
$$

is invertible and clearly takes voltages $\zeta_{C_{i}}^{U \oplus W}$ to voltages $\zeta_{C_{i}}^{U^{\prime} \oplus W^{\prime}}$. Hence the restrictions of $\zeta^{U \oplus W}$ and $\zeta^{U^{\prime} \oplus W^{\prime}}$ to $X$ are equivalent. 
In order to test which 2-dimensional subspaces give rise to connected and possibly equivalent coverings of $X$ we only need to check, by Lemma 4.1, the subspaces $\mathcal{L}_{A}(i)$, $\mathcal{L}_{A}(-i)$, and the following direct sums:

$$
\mathcal{L}_{A}(1) \oplus W_{0}(i), \mathcal{L}_{A}(1) \oplus W_{0}(-i), W_{0}(i) \oplus W_{0}(-i)
$$

First note that the two subspaces $\mathcal{L}_{A}(i)$ and $\mathcal{L}_{A}(-i)$ give rise to disconnected covers of $X$ since each can be written as a direct sum of two 1-dimensional subspaces, of which one gives rise to a disconnected cover of $X$. As for the remaining subspaces, they all give rise to connected and pairwise nonequivalent coverings of $X$. We leave this to the reader.

Finally, any 3-dimensional subspace giving rise to a connected cover of $X$ is equivalent to the homological cover over $X$. So it is enough to identify one such subspace. The reader can check that $\mathcal{L}_{A}(1) \oplus W_{0}(i) \oplus W_{0}(-i)$ satisfies the connectedness condition. This completes the analysis when $p$ is odd.

Case $p=2$.

In this case the representation of the group $\langle A\rangle$ is not completely reducible. First we need an appropriate Jordan basis for the matrix $A$. Observe that the respective Jordan form has two elementary Jordan matrices, one of size 4 and one of size 2. By computation, a Jordan basis is, say,

$$
\begin{aligned}
& v_{1}=(1,1,1,1,0,0)^{t}, \\
& b_{1}=(0,1,0,1,0,0)^{t}, \\
& b_{3}=(0,0,1,1,0,0)^{t}, \\
& b_{4}=(0,0,0,1,0,0)^{t}, \\
& v_{2}=(0,0,0,0,1,1)^{t}, \\
& b_{2}=(0,0,0,0,1,0)^{t},
\end{aligned}
$$

where $v_{1}$ and $v_{2}$ are the eigenvectors, the 4-dimensional cyclic subspace is spanned by $v_{1}, b_{1}, b_{3}, b_{4}$, and the 2 -dimensional one by $v_{2}, b_{2}$.

There are exactly three 1 -dimensional $A$-invariant subspaces, all contained in the 2 dimensional eigenspace $\mathcal{L}_{A}(1)$, namely $W_{\infty}(1)=\left\langle v_{1}\right\rangle, W_{0}(1)=\left\langle v_{2}\right\rangle$ and $W_{1}(1)=$ $\left\langle(1,1,1,1,1,1)^{t}\right\rangle$. Only the latter two give rise to connected covers of $X$. Moreover, both also give rise to equivalent coverings of $X$. As a representative we choose, say, $W_{1}(1)$. The resulting cover is the canonical double cover.

As for the 2-dimensional $A$-invariant subspaces, there are exactly seven of them. One is the eigenspace $\mathcal{L}_{A}(1)=\left\langle v_{1}, v_{2}\right\rangle$. The other six subspaces arise from vectors $u \in$ $\operatorname{Ker}(A-I)^{2} \backslash \mathcal{L}_{A}(1)$. Such a 2-dimensional subspace consists of the following vectors: $0, u, A u, u+A u$. Clearly $A u \neq u$ (since $u$ is not an eigenvector), and $A u \in \operatorname{Ker}(A-I)^{2} \backslash$ $\mathcal{L}_{A}(1)$ (as $A^{2} u=A u$ implies $A u=u$ ). Therefore the elements in $\operatorname{Ker}(A-I)^{2} \backslash \mathcal{L}_{A}(1)$ in the same 2 -dimensional subspace come in pairs. As the set $\operatorname{Ker}(A-I)^{2} \backslash \mathcal{L}_{A}(1)$ contains exactly 12 nontrivial vectors, there are exactly six subspaces of this kind. These can be explicitly represented as $\left\langle v_{1}, b_{1}\right\rangle,\left\langle v_{1}, u_{1}\right\rangle,\left\langle v_{2}, b_{2}\right\rangle,\left\langle v_{2}, u_{2}\right\rangle,\left\langle v_{1}+v_{2}, u_{3}\right\rangle,\left\langle v_{1}+v_{2}, u_{4}\right\rangle$, 
where

$$
\begin{aligned}
& u_{1}=(0,1,0,1,1,1)^{t}, \\
& u_{1}=(1,1,1,1,1,0)^{t}, \\
& u_{3}=(1,0,1,0,0,1)^{t}, \\
& u_{4}=(1,0,1,0,1,0)^{t} .
\end{aligned}
$$

The reader can check that the subspaces giving rise to connected covers of $X$ are pairwise equivalent. As a representative we choose, say, $\left\langle v_{2}, b_{2}\right\rangle$.

Consider now the 3 -dimensional $A$-invariant subspaces. It is enough to find just one (if it exists) giving rise to a connected cover of $X$ (which is then equivalent to the homological cover of $X$ ). However, the reader can check that all 3-dimensional subspaces give rise to disconnected covers of $X$. To this end we only provide a basis for each of them. Note that there are seven 3 -dimensional subspaces in all. Indeed, three such subspaces exist in $\operatorname{Ker}(A-I)^{2}$, namely

$$
\left\langle v_{1}, v_{2}, b_{1}\right\rangle,\left\langle v_{1}, v_{2}, b_{2}\right\rangle \text {, and }\left\langle v_{1}, v_{2}, u_{4}\right\rangle,
$$

each containing $\mathcal{L}_{A}(1)$. The other four arise as cyclic subspaces of the Jordan chains of length 3 (note that there are 16 chains in all, and $A$ acts semi-regularly on the set of vectors in $\operatorname{Ker}(A-I)^{3} \backslash \operatorname{Ker}(A-I)^{2}$ with four orbits of size 4$)$. The respective bases are $\left\{v_{1}, b_{1}, b_{3}\right\},\left\{v_{1}, b_{1}, u_{5}\right\},\left\{v_{1}, u_{6}, u_{7}\right\},\left\{v_{1}, u_{6}, u_{8}\right\}$, where

$$
\begin{aligned}
& u_{5}=(0,0,1,1,1,1)^{t}, \\
& u_{6}=(1,0,1,0,1,1)^{t}, \\
& u_{7}=(1,0,0,1,0,1)^{t}, \\
& u_{8}=(1,0,0,1,1,0)^{t} .
\end{aligned}
$$

This completes the analysis for $p=2$.

Remark 4.2. In order to further reduce these coverings up to isomorphism we can follow (ii) of Theorem 2.1. The possibility that the projections in Table 1 are isomorphic is converted to checking the pairs in rows $2,3,4$ and those in rows $7,8,9$. The reader can check that rows 3 and 4 give rise to isomorphic covers as well as rows 7 and 8 .

Remark 4.3. Consider the automorphism $h=(12)$ of $X$. Clearly, $g$ and $h$ generate the full automorphism group $\operatorname{Aut}(X)$. Let $\left(h^{*}\right)^{\#}$ be the linear transformation of $H_{1}\left(\widehat{X}(\Omega) ; \mathbb{Z}_{p}\right)$ induced by the natural action of $h^{*}$ on $H_{1}\left(\widehat{X}(\Omega) ; \mathbb{Z}_{p}\right)$, and let $M_{h^{*}} \in \mathbb{Z}_{p}^{6,6}$ be its matrix representation with respect to the basis $\mathcal{B}_{\mathcal{T}^{*}}$. By computation we have that

$$
M_{h^{*}}^{t}=\left[\begin{array}{cccccc}
-1 & 0 & 0 & 0 & 0 & 0 \\
0 & 0 & 0 & 0 & 0 & -1 \\
0 & 0 & 1 & 0 & 0 & 0 \\
0 & 0 & 0 & 1 & 0 & 0 \\
0 & 0 & 0 & -1 & 0 & 0 \\
0 & -1 & 0 & 0 & 0 & 0
\end{array}\right] .
$$

It is now easy to check that among subspaces in Table 1 only $W_{1}(1)$ is also $M_{h^{*}}^{t}$-invariant. Thus, the canonical double cover of $X$ is the only covering along which the full automorphism group $\operatorname{Aut}(X)$ lifts as a sectional split extension over $\Omega$. 
Table 1: All voltage assignments on $K_{4}$ giving rise to pairwise nonequivalent connected elementary abelian regular covering projections along which the cyclic group $\langle g\rangle$ of automorphisms of $K_{4}$ lifts as a sectional split extension. Additionally, coverings in rows 3 and 4 are isomorphic, as well as those in rows 7 and 8 .

\begin{tabular}{|c|c|c|c|c|c|c|c|c|}
\hline$n$ & Inv. subsp. & $\zeta_{x_{1}}$ & $\zeta_{x_{2}}$ & $\zeta_{x_{3}}$ & $\zeta_{x_{4}}$ & $\zeta_{x_{5}}$ & $\zeta_{x_{6}}$ & Condition \\
\hline 1 & $W_{1}(1)$ & {$[1]$} & {$[1]$} & {$[1]$} & {$[1]$} & {$[1]$} & {$[1]$} & $p=2$ \\
\hline 2 & $\left\langle v_{1}\right\rangle$ & {$[1]$} & {$[1]$} & {$[1]$} & {$[1]$} & {$[0]$} & {$[0]$} & $p \neq 2$ \\
\hline 3 & $\left\langle v_{i}\right\rangle$ & {$[1]$} & {$[i]$} & {$[-1]$} & {$[-i]$} & {$[0]$} & {$[0]$} & $\begin{array}{c}p \equiv 1(\bmod 4) \\
i^{2}=-1\end{array}$ \\
\hline 4 & $\left\langle v_{-i}\right\rangle$ & {$[1]$} & {$[-i]$} & {$[-1]$} & {$[i]$} & {$[0]$} & {$[0]$} & $\begin{array}{c}p \equiv 1(\bmod 4) \\
i^{2}=-1\end{array}$ \\
\hline 5 & $\left\langle v_{2}, b_{2}\right\rangle$ & {$\left[\begin{array}{l}0 \\
0\end{array}\right]$} & {$\left[\begin{array}{l}0 \\
0\end{array}\right]$} & {$\left[\begin{array}{l}0 \\
0\end{array}\right]$} & {$\left[\begin{array}{l}0 \\
0\end{array}\right]$} & {$\left[\begin{array}{l}1 \\
1\end{array}\right]$} & {$\left[\begin{array}{l}1 \\
0\end{array}\right]$} & $p=2$ \\
\hline 6 & $W_{1,1,0,0}$ & {$\left[\begin{array}{l}1 \\
1\end{array}\right]$} & {$\left[\begin{array}{c}1 \\
-1\end{array}\right]$} & {$\left[\begin{array}{l}-1 \\
-1\end{array}\right]$} & {$\left[\begin{array}{c}-1 \\
1\end{array}\right]$} & {$\left[\begin{array}{l}0 \\
0\end{array}\right]$} & {$\left[\begin{array}{l}0 \\
0\end{array}\right]$} & $p \equiv 3(\bmod 4)$ \\
\hline 7 & $\left\langle v_{1}, v_{i}\right\rangle$ & {$\left[\begin{array}{l}1 \\
1\end{array}\right]$} & {$\left[\begin{array}{l}1 \\
i\end{array}\right]$} & {$\left[\begin{array}{c}1 \\
-1\end{array}\right]$} & {$\left[\begin{array}{c}1 \\
-i\end{array}\right]$} & {$\left[\begin{array}{l}0 \\
0\end{array}\right]$} & {$\left[\begin{array}{l}0 \\
0\end{array}\right]$} & $\begin{array}{c}p \equiv 1(\bmod 4) \\
i^{2}=-1\end{array}$ \\
\hline 8 & $\left\langle v_{1}, v_{-i}\right\rangle$ & {$\left[\begin{array}{l}1 \\
1\end{array}\right]$} & {$\left[\begin{array}{c}1 \\
-i\end{array}\right]$} & {$\left[\begin{array}{c}1 \\
-1\end{array}\right]$} & {$\left[\begin{array}{l}1 \\
i\end{array}\right]$} & {$\left[\begin{array}{l}0 \\
0\end{array}\right]$} & {$\left[\begin{array}{l}0 \\
0\end{array}\right]$} & $\begin{array}{c}p \equiv 1(\bmod 4) \\
i^{2}=-1\end{array}$ \\
\hline 9 & $\left\langle v_{i}, v_{-i}\right\rangle$ & {$\left[\begin{array}{l}1 \\
1\end{array}\right]$} & {$\left[\begin{array}{c}i \\
-i\end{array}\right]$} & {$\left[\begin{array}{l}-1 \\
-1\end{array}\right]$} & {$\left[\begin{array}{c}-i \\
i\end{array}\right]$} & {$\left[\begin{array}{l}0 \\
0\end{array}\right]$} & {$\left[\begin{array}{l}0 \\
0\end{array}\right]$} & $\begin{array}{c}p \equiv 1(\bmod 4) \\
i^{2}=-1\end{array}$ \\
\hline 10 & $\left\langle v_{1}, W_{1,1,0,0}\right\rangle$ & {$\left[\begin{array}{l}1 \\
1 \\
1\end{array}\right]$} & {$\left[\begin{array}{c}1 \\
1 \\
-1\end{array}\right]$} & {$\left[\begin{array}{c}1 \\
-1 \\
-1\end{array}\right]$} & {$\left[\begin{array}{c}1 \\
-1 \\
1\end{array}\right]$} & {$\left[\begin{array}{l}0 \\
0 \\
0\end{array}\right]$} & {$\left[\begin{array}{l}0 \\
0 \\
0\end{array}\right]$} & $p \equiv 3(\bmod 4)$ \\
\hline 11 & $\left\langle v_{1}, v_{i}, v_{-i}\right\rangle$ & {$\left[\begin{array}{l}1 \\
1 \\
1\end{array}\right]$} & {$\left[\begin{array}{c}1 \\
i \\
-i\end{array}\right]$} & {$\left[\begin{array}{c}1 \\
-1 \\
-1\end{array}\right]$} & {$\left[\begin{array}{c}1 \\
-i \\
i\end{array}\right]$} & {$\left[\begin{array}{l}0 \\
0 \\
0\end{array}\right]$} & {$\left[\begin{array}{l}0 \\
0 \\
0\end{array}\right]$} & $\begin{array}{c}p \equiv 1(\bmod 4) \\
i^{2}=-1\end{array}$ \\
\hline
\end{tabular}

Acknowledgement. The author would like to thank Aleksander Malnič for enlightening discussions, and both referees for their helpful suggestions that improved the presentation.

\section{References}

[1] N. L. Biggs, Algebraic Graph Theory, Cambridge Univ. Press, Cambridge, 1974.

[2] S. J. Curran, D. W. Morris and J. Morris, Cayley graphs of order $16 p$ are hamiltonian, Ars Math. Contemp. 5 (2012), 189-215.

[3] S. F. Du, J. H. Kwak and M. Y. Xu, Lifting of automorphisms on the elementary abelian regular coverings, Lin. Alg. Appl., 373 (2003), 101-119.

[4] Y. Q. Feng and J. H. Kwak, s-regular cubic graphs as coverings of the complete bipartite graph $K_{3,3}$, J. Graph Theory 45 (2004), 101-112.

[5] Y. Q. Feng and J. H. Kwak, Classifying cubic symmetric graphs of order $10 p$ or $10 p^{2}$, Science China Ser. A: Math. 49 (2006), 300-319.

[6] Y. Q. Feng and J. H. Kwak, Cubic symmetric graphs of order a small number times a prime or a prime square, J. Combin. Theory Ser. B 97 (2007), 627-646. 
[7] Y. Q. Feng, J. H. Kwak and K. Wang, Classifying cubic symmetric graphs of order $8 p$ or $8 p^{2}$, Europ. J. Combin. 26 (2005), 1033-1052.

[8] Y. Q. Feng, A. Malnič, D. Marušič and K. Kutnar, On 2-fold covers of graphs, J. Combin. Theory Ser. B 98 (2008), 324-341.

[9] J. L. Gross and T. W. Tucker, Topological Graph Theory, Wiley - Interscience, New York, 1987.

[10] N. Jacobson, Lectures in Abstract Algebra, II. Linear Algebra, Springer, New York, 1953.

[11] M. Klin and C. Pech, A new construction of antipodal distance regular covers of complete graphs through the use of Godsil-Hensel matrices, Ars Math. Contemp. 4 (2011), 205-243.

[12] K. Kutnar, D. Marušič, D. W. Morris, J. Morris and P. Šparl, Hamiltonian cycles in Cayley graphs whose order has few prime factors, Ars Math. Contemp. 5 (2012), 27-71.

[13] B. Kuzman, Arc-transitive elementary abelian covers of the complete graph $K_{5}$, Linear Algebra Appl. 433 (2010), 1909-1921.

[14] J. H. Kwak and J. M. Oh, Arc-transitive elementary abelian covers of the octahedron graph, Linear Algebra Appl. 429 (2009), 2180-2198.

[15] A. Malnič, Group actions, coverings and lifts of automorphisms, Discrete Math. 182 (1998), 203-218.

[16] A. Malnič, R. Nedela and M. Škoviera, Lifting graph automorphisms by voltage assignments, European J. Combin. 21 (2000), 927-947.

[17] A. Malnič, Action graphs and coverings, Discrete Math. 224 (2002), 299-322.

[18] A. Malnič, D. Marušič, S. Miklavič and P. Potočnik, Semisymmetric elementary abelian covers of the Möbius-Kantor graph, Discrete Math. 307 (2007), 2156-2175.

[19] A. Malnič, D. Marušič and P. Potočnik, Elementary abelian covers of graphs, J. Alg. Combin. 20 (2004), 71-96.

[20] A. Malnič, D. Marušič and P. Potočnik, On cubic graphs admitting an edge-transitive solvable group, J. Algebraic Combin. 20 (2004), 99-113.

[21] A. Malnič and P. Potočnik, Invariant subspaces, duality, and covers of the Petersen graph, European J. Combin. 27 (2006), 971-989.

[22] A. Malnič and R. Požar, On the Split Structure of Lifted Groups, submitted.

[23] A. Malnič and R. Požar, On the Split Liftings with Sectional Complements, in preparation.

[24] J. M. Oh, A classification of cubic s-regular graphs of order 14p, Discrete Math. 309 (2009), 2721-2726.

[25] J. M. Oh, Arc-transitive elementary abelian covers of the Pappus graph, Discrete Math. 309 (2009), 6590-6611.

[26] J. Širáň, Coverings of graphs and maps, ortogonality, and eigenvectors, J. Algebraic Combin. 14 (2001), 57-72.

[27] M. Škoviera, A contribution to the theory of voltage graphs, Discrete Math. 61 (1986), 281292. 